\title{
LOS ESPACIOS OLVIDADOS DE PARTICIPACIÓN Y AUTONOMÍA ESCOLAR FRENTE A LOS PROCESOS DE RENDICIÓN DE CUENTAS Y BUROCRACIA EN MÉXICO
}

\section{THE FORGOTTEN SPACES OF PARTICIPATION AND SCHOOL AUTONOMY IN THE FACE OF ACCOUNTABILITY AND BUREAUCRACY PROCESSES IN MEXICO}

\author{
Haleyda Quiroz Reyes
}

\section{ABSTRACT}

This article reflects on the dilemma presented by international discourses on an educational renewal that is committed to granting autonomy to schools as nerve centers where true pedagogical action spaces are developed as opposed to the demands for obtaining successful test results, global comparisons and accountability. It is in this contradictory overwhelming and inoperative challenge of education where we intend to focus this analysis that we approach with a positive look at progressive autonomy and its struggle to adapt to the particularities and needs of the social and cultural context of schools under the influence of a bureaucratic context. In the case of Mexico, facing two important challenges, getting rid of bureaucratic structures and in turn responding to the demands of transparency and accountability that hinder pedagogical initiatives and participation of school management under evaluation processes with inquisitive requirements designed and programmed from the central administration. However, there is an inexhaustible effort to advance in the challenge of reestablishing spaces for democratic and collaborative participation where educational purposes can once again be linked to the aspirations, goals and daily life of the school community.

Key words: school autonomy, participation, school management, bureaucracy, accountability.

\section{RESUMEN}

Este articulo reflexiona en la disyuntiva que presentan los discursos internacionales sobre una renovación educativa que apuesta por conceder autonomía a las escuelas como centros neurálgicos en donde se desarrollan los verdaderos espacios de acción pedagógica, en contraposición con las exigencias por la obtención de resultados exitosos en exámenes, comparativas globales y rendición de cuentas. Es en este reto contradictorio, agobiante e inoperante de la educación donde pretendemos centrar este análisis que abordamos con una mirada positiva a la autonomía progresista y su lucha por adaptarse a las particularidades y necesidades del contexto social y cultural de las escuelas, bajo el influjo de un contexto burocrático. En el caso de México, enfrentándose a dos retos importantes, deshacerse de estructuras burocráticas y a su vez responder a las exigencias de transparencia y rendición de cuentas que obstaculizan las iniciativas pedagógicas y de participación de la gestión escolar bajo procesos evaluativos con requerimientos inquisitivos diseñados y programados desde la cúpula central. No obstante, existe un inagotable esfuerzo por avanzar en el desafío de reestablecer los espacios y canales de participación democrática y colaborativa, en donde nuevamente se puedan enlazar los propósitos educativos con las aspiraciones, metas y vida cotidiana de la comunidad escolar. 
Los espacios olvidados de participación y autonomía escolar frente a los procesos de rendición de cuentas y burocracia en México Haleyda Quiroz Reyes JOURNAL OF SUPRANATIONAL POLICIES OF EDUCATION, nº11, pp. 87-105 DOI: https://doi.org/10.15366/jospoe2020.11.005

Palabras clave: autonomía escolar, participación, gestión escolar, burocracia, rendición de cuentas.

Fecha de recepción: 22 de abril de 2020.

Fecha de aceptación: 25 de septiembre de 2020. 


\section{INTRODUCCIÓN}

Los países han realizado grandes esfuerzos de descentralización en el ámbito de la gestión escolar, con el propósito de alcanzar mejoras en la calidad educativa. La búsqueda ansiosa por permanecer en una carrera global de resultados competitivos para poder incorporarse en la lista de sistemas educativos exitosos, es uno de los principales alicientes que dan dirección a políticas y reformas educativas en los últimos años. Dentro de este paraíso de atracción mundial, la autonomía representa uno de los elementos centrales de algunos países con prácticas educativas eficaces.

Este fenómeno conlleva que las escuelas, no se centran en mirar con atención y profundidad la raíz de sus problemas educativos y muncho menos en el qué y para qué de la propia educación que ellos ofrecen. Aún más, no miran las propuestas de solución de una escuela de la zona aledaña o de otro municipio, condado o comunidad con características similares. Actualmente, la tendencia de las miradas se centra en los países que han demostrado estadísticamente ser exitosos como Finlandia, Macao, Países Bajos o Canadá. La atracción es a nivel mundial en los sistemas educativos y paulatinamente ha dejado de ser local, aunque dentro de estos contextos ya existían diferentes proceso participativos olvidados como la autonomía escolar, que irrumpe nuevamente en las recomendaciones mundiales por ser un elemento importante en esas potencias internacionales.

\section{LA AUTONOMÍA ESCOLAR, UNA UTOPÍA O UNA REALIDAD ASEQUIBLE.}

Vivimos en un contexto global en dónde se está produciendo una extraordinaria expansión en la difusión de las recomendaciones, análisis y discursos bajo el amparo de la eficacia y eficiencia, la calidad, la evaluación, los resultados efectivos y la competitividad. Detrás de la conformación de esa gramática global, se encuentran organizaciones internacionales que son consideradas especialistas en el ámbito de las cuestiones educativas. Dentro de estos actores principales, se encuentra la OCDE, un experto en estudiar, diagnosticar y diseñar lineamientos de acción para mejorar los sistemas educativos, pero también con una capacidad formidable para "concretar una uniformidad que margina y / o socava las voces de los principales interesados, como los docentes y los investigadores en educación” (Wiseman y Stevens, 2017, p.259).

La autonomía escolar, no es la excepción y la OCDE (2015), la recomienda de manera global, como estrategia que influye positivamente en el desempeño de las escuelas. Apuesta por profesionalizar a los líderes educativos y, exigirles que rindan cuentas, que participen en las decisiones fundamentales de sus escuelas, tales como contratar o despedir docentes, así como en establecer estructuras de decisión adaptadas a sus contextos para tener un impacto positivo.

La autonomía es valorada, por ser un elemento presente en los países que han obtenido resultados exitosos. De esta manera ha renacido, incluso dentro de los servicios públicos, cambiando el rumbo de muchas decisiones que son basadas en estos principios imprescindibles "como la autonomía escolar, la rendición de cuentas, la gestión basada en resultados o la libertad de elección escolar y, que han penetrado profundamente en cómo se regulan, proveen y financian los servicios educativos" (Normad y Verger, 2015, p.600).

Este nuevo panorama de competencia planetaria en la gestión y administración pública, para Rizvi y Lingard (2013), es el responsable de la transformación de una nueva posición de los 
estados, que se ven cada vez más vulnerados en su autonomía y autoridad frente a los imperativos de la economía global, que indiscutiblemente repercute en los beneficios que este le brinda a la sociedad y sus decisiones en términos de políticas públicas. Sin embargo, aún existen mediaciones desde los niveles locales y regionales, donde esos intereses parecen encontrar un límite entre esas decisiones globales y las locales.

Es por esta razón, que nos parece relevante. rescatar ciertos aspectos de la autonomía que pueden representar una serie de oportunidades y ventajas para los sistemas educativos. En primera instancia, las políticas públicas centrales tendrían que iniciar por otorgar a los gobiernos locales un lugar preponderante en la realización e integración de sus acciones locales y no ser simples ejecutores de prácticas globales, otorgando "capacidad propia para definir y dotar políticas públicas, para lo que resulta imperativo el reconocimiento de un ámbito propio de autonomía, dentro del cual puedan ejercer su papel sin intromisiones ni tutelas" (Spink, Longo, Echebarría y Stark, 2001, p.138). Precisamente dentro de este principio, se encuentra uno de los propósitos de este estudio, que es, profundizar en la autonomía escolar desde su vertiente mas positiva, constructiva, social y tradicional que ha estado en las acciones de algunos países desde antes de penetrar en la retórica global.

Macri (2002), distingue cuatro tipos de autonomía para las instituciones escolares: financiera, de gestión, organizativa y didáctica. Y en conjunto, la autonomía se expresa en la identidad de la institución, la toma de decisiones, la estructura y la estrategia. A su vez, se ejerce en ámbitos como el diseño de los contenidos y estrategias didácticas y pedagógicas; la administración de la institución; las políticas y el gobierno; la gestión de los recursos humanos, y los servicios ofrecidos por la institución. Hablamos de una autonomía integral y que se desarrolla desde el centro neurálgico de los sistemas educativos que son las escuelas en un amplio equilibrio entre acciones académicas y administrativas.

Si analizamos la construcción de la autonomía desde esta perspectiva escolar integradora, es indispensable categorizarla como lo hacen Normad y Verger (2015) en dos vertientes, la autonomía de gestión y la autonomía pedagógica. Ambas, le brindan a los centros escolares la capacidad de decisión sobre el proyecto educativo, los contenidos curriculares y los sistemas de evaluación, promoviendo que la educación se adapte a las particularidades del contexto social y cultural y, tiene como principios: la profesionalización, la renovación pedagógica y la atención a la diversidad.

El reto es centrarse en un denominador común, el centro educativo y sus necesidades. Esto nos lleva directamente a encaminarnos hacia un análisis de la autonomía escolar progresista con "un uso más flexible del tiempo, favorecer el sentido de la colegialidad, una mayor cultura profesional, expectativas más elevadas y ampliación del papel del profesor, al que se le debe mayor reconocimiento y estatus y, un sistema de incentivos que favorezca el desarrollo profesional y el aprendizaje" (Davis y Thomas, 1992, p.12).

Sin pretender agotar el tema, es importante no olvidar la conexión intrínseca entre la autonomía institucional y las normas, reglamentos y los procesos administrativos propios de las instituciones escolares. Macri (2002) reflexiona sobre esta relación y concluye que, es posible inferir que será baja la autonomía institucional cuando la presión normativa (leyes, decretos, reglamentos) es alta, en estos casos la uniformidad escolar es abundante. Si por el contrario la normativa es escasa la autonomía es más amplia y los centros escolares serán más responsables en cuanto a resultados y podrá desarrollarse un ambiente de compromiso colectivo al no trabajar bajo exigencias externas. 
En suma, una autonomía progresista de los centros escolares, hace énfasis en dos herramientas fundamentales para lograr ese trabajo y compromiso colaborativo, en donde la autonomía escolar verdaderamente facilite los espacios para la organización de las acciones y contenidos educativos. En primera instancia esta el proyecto escolar que representa el elemento que otorga orden dentro de la gestión, siempre y cuando se realice en un sentido amplio que va desde el ámbito organizativo, pedagógico y de contenidos. En segundo plano, esta "la evaluación, visto como un principio de transparencia, que proporciona información para mejorar la calidad, eficiencia y equidad del sistema educativo a través de los agentes implicados en el proceso" (Blanch, 2011, p. 21).

\section{LA SOMBRA DE LA BUROCRACIA COMO LIMITANTE PARA LA AUTONOMÍA ESCOLAR.}

Como lo hemos reflexionado en el apartado anterior, hoy somos testigos de un número considerable de países que están implementando y llevando a cabo numerosos cambios y reformas en sus sistemas educativos. Esto responde a la idea introducida por Rizvi y Lingard (2013) sobre una política que, en ocasiones es una expresión de los efectos específicos de la globalización. Pero también se puede percibir como la forma en que el Estado y los políticos aspiran a gestionar y re articular las presiones globales, para equilibrarlas frente a las demandas locales y nacionales de los intereses competitivos.

En este caso, la gobernabilidad en la gestión del sistema educativo se distingue tanto por la búsqueda de la estabilidad como por la eficiencia y la eficacia gubernamental. Por ello, para su modernización, la gestión de los sistemas educativos y, específicamente en el caso mexicano ha adoptado los postulados y herramientas de la Nueva Gestión Pública (NGP). De este modo responde y se adapta a los cambios impulsados por las grandes tendencias internacionales, disponiendo así de una legitimidad sustentada no en un acuerdo de políticas educativas locales, sino en pactos políticos respaldados por discursos globales.

México no es la excepción en emprender nuevos esfuerzos por entrar en ese camino global de sistemas educativos competitivos, en busca de la calidad o ser exitosos. "La respuesta de México al desafío de su sistema educativo nacional frente a esos temas, ha sido una apuesta estratégica gubernamental que contempla simultáneamente la modernización de la gestión del sistema cuyo diseño y propósito responde al paradigma de la NGP; y la apuesta por la gestión basada en la escuela, como parte de la adopción del movimiento de escuelas eficaces" (Del Castillo, 2012, p.164).

Para estos fines de innovación y progreso mundial, en la educación básica en México durante el período 1994 - 2011, se implementaron acciones que comprenden programas sectoriales de educación pública, políticas de formación para la gestión de directivos de centros escolares (jefes de enseñanza, inspectores, supervisores, asesores técnico pedagógicos, directores y subdirectores de escuelas) que han ido adquiriendo cada vez más una creciente importancia como figura de liderazgo y cambio. El reflejo de este interés se proyectó en establecer el programa escuelas de calidad, acompañado por la creación de una dependencia especifica para la organización y la gestión, con la finalidad de fortalecer la gobernanza escolar con la ayuda de los lideres educativos.

Posteriormente y como sucedió en muchos países, desde el ciclo escolar 2001-2002, México apuesta por el término calidad. Así que entra en vigor el Programa Escuelas de Calidad, el cual incorpora un modelo de autogestión basada en principios democráticos que ubican a la escuela 
como centro de toda iniciativa del sistema educativo. Su objetivo general se encontraba, en lograr una mayor calidad de los resultados educativos, a partir de la transformación de la gestión de las escuelas, promoviendo la construcción de un modelo de autogestión basado en la capacidad de toma de decisiones. Fortaleciendo de esta manera el liderazgo compartido, el trabajo en equipo, la participación social responsable, las prácticas docentes más flexibles que atiendan a la diversidad de los alumnos; una gestión basada en la evaluación para la mejora continua y la planeación participativa, impulsando la innovación educativa.

Así, México se suma al ideal de escuelas que se auto-gestionan y que son lideradas de forma colaborativa por la figura enaltecida de los directivos escolares. En esta búsqueda por mejorar la calidad del servicio educativo y la gestión escolar, a partir del ciclo escolar 2002-2003 se dieron a conocer las Reglas de Operación del Programa y los estándares de gestión escolar, con el fin de orientar los desempeños deseables en una escuela de calidad, que en 2019 es sustituida por el de escuela exitosa.

Esta pequeña pincelada sobre la entrada del sistema educativo a la corriente de competitividad global, nos lleva fundamentalmente a dos reflexiones. La primera que desarrollaremos más adelante, con referencia a los espacios de participación local que estas políticas promueven y restringen. Y la segunda que a continuación planteamos, que es la referente a la forma de entender la aplicación de estas acciones que son sumamente innovadoras y sobre todo esperanzadoras en diferentes ámbitos, uno de ellos en redimir la figura central de las escuelas como el espacio preponderante para el desarrollo de iniciativas educativas mediante la participación de los actores principales profesores, personal directivo, padres de familia y comunidad y bajo principios democráticos que conformen una autogestión de forma colaborativa. Desde esta perspectiva de gobernanza escolar, podríamos ser optimistas, sin embargo la pregunta principal es ¿qué sucede cuando estos elementos se encuentran bajo fuertes estructuras de burocracia administrativa?

Dentro de los discursos globales que exigen la resolución de los problemas y retos que debe emprender la educación a nivel mundial, se hace el llamado a que todos y cada uno de los países, tanto los desarrollados como los que se encuentran en vías de desarrollo se encaminen hacia la senda del progreso. Pero se olvida que cada país tiene un contexto social, cultural, histórico, administrativo, económico y político totalmente diferentes y por tanto, la forma de afrontar esos desafíos no podrá de ninguna manera ser uniforme.

Específicamente en el caso de las administraciones públicas en América Latina, se instala la NGP, bajo instituciones pre-burocráticas en las que prevalecen el clientelismo, la inseguridad jurídica y la corrupción. Por tanto, el control, seguimiento y la evaluación de los servicios externalizados son acciones complicadas y económicamente costosas de realizar. Primordialmente porque hablamos de estructuras organizativas jóvenes en términos históricos de descentralización. Ante esta realidad, las consultorías internacionales aparecen como la mejor opción, aunque a estas "no le preocupan los problemas autóctonos -incluso los niega: todas las organizaciones tienen idénticos problemas con independencia de que sean privadas o públicas y con independencia de su contexto económico y social, es su base gubernamental-" (Ramió y Salvador, 2005, p.36).

De esta manera, tanto México como los países de América Latina y el Caribe forman parte de la financiación del Banco Mundial (Verger y Normad, 2015) para facilitar su entrada en ese modelo global de descentralización en los años 90's. Sustentado por la debilidad institucional de sus administraciones, su falta para regular de forma eficiente y con neutralidad los ámbitos públicos 
y privados y por "no prestar servicios públicos adecuados a las necesidades existentes; ni redistribuir correctamente los recursos para alcanzar una mayor equidad social" (Barzelay, 2003, p.12).

México, por su parte, se caracteriza por un sistema tradicionalmente burocrático y clientelar mediado por la corrupción en todo su sistema de administración pública. Es verdad, que aún no han sufrido una total inserción al mercado privado, por continuar con un Estado asistencialista que se resiste a la privatización o concertación del sistema educativo, que continua siendo respaldado por sindicatos de maestros y bajo un lema de justicia social que han limitado las acciones radicales de descentralización en términos de mercantilización de la escuela.

El perspicaz estudio de Egido, Álvarez y Figueiredo (2000), ratifica como la autonomía financiera de México esta ligada a un proceso intenso de burocratización, quedando evidenciado con tres variables que inciden directamente sobre el control externo del manejo del presupuesto. La primera es que los centros educativos no deciden sobre sus recursos financieros. La segunda se refiere a que los directivos solo administran los recursos y dan cuenta de estos bajo una normativa excesivamente burocratizada. Por último, existe una cooperativa escolar pero con licitación estatal. Sólo existe un manejo de ciertos presupuestos provenientes de la SEP (Secretaria de Educación Pública) con autonomía vigilada para gestionarlos.

Ante este desafío se ha optado por la descentralización de los servicios con la finalidad de terminar con la burocracia que ha caracterizado a las estructuras públicas durante años. Pero es una carga pesada que trabaja en contraposición de las nuevas exigencias. Albi, González-Páramo y Casanovas (1997), recomiendan las unidades gestionables a través de la medición y la evaluación de los resultados de la gestión con la finalidad de encontrar esa eficiencia perdida durante muchos años, "lograr que los gestores públicos sirvan a los ciudadanos, ayuda, evidentemente a mejorar el sector público, siempre que no se cometa el error de creer que la administración pública y una organización empresarial son exactamente la misma cosa” (p. 176).

Indudablemente, pasar de la administración tradicional -con su gran dosis de burocracia- a la gestión pública eficiente, requieren esfuerzos organizativos y sobre todo una planeación estratégica en la toma de decisiones. En este sentido, la disyuntiva entre Estado o mercado rodea no sólo los discursos sino también está presente en las reformas públicas y en su aplicación. Pero, las instancias administrativas en México, nunca se han caracterizado por llevar con eficacia los aspectos normativos de los programas educativos, al contrario lamentablemente, se han distinguido -y no sólo en el ámbito educativo- por presentar una burocracia que limita el manejo y modernización de los sistemas de gestión. Es entendible por tanto, que uno de los principales propósitos del país sea lo que Egido et al. (2000) llaman una tendencia a desburocratizar el aparato central, buscando así mayor eficiencia y racionalidad en el manejo y generación de los recursos financieros. Lo que se espera con la descentralización además de ganar eficiencia administrativa en el manejo de los fondos, es que los entes responsables asuman la tarea de buscar alternativas para la consecución de recursos complementarios. Astiz (2015), confirma esta postura:

la autogestión escolar en un contexto de aplicación global de prácticas de descentralización, no siempre constituye un proceso universal, deseado y posible, tal como se lo presenta, ni tampoco contribuye en todos los casos al fortalecimiento de prácticas democráticas en la educación y en la sociedad civil. Por el contrario, constituye un proceso complejo que involucra diferentes sectores del Estado y de la sociedad y depende de un aún más complejo grupo de factores organizacionales y sociopolíticos. (p.667) 
Con esta reflexión deseamos resaltar la influencia de factores administrativos y organizativos de las estructuras de gestión en su intervención en el éxito escolar, para este fin Alvariño, Arzola, Brunner, Recart y Vizcarra (2000), subrayan la importante incidencia en una buena gestión del clima organizacional que afecta en las formas de liderazgo y conducción institucionales, en el aprovechamiento óptimo de los recursos humanos y del tiempo, en la planificación de tareas y la distribución del trabajo y su productividad, también en la eficiencia de la administración y el rendimiento de los recursos materiales y por tanto en la calidad de los procesos educacionales.

Si analizamos esos elementos, encontramos que plantean una visión de la gestión focalizada en la movilización de recursos. Desde esta perspectiva, podríamos entender la gestión en términos de capacidad. La gestión puede generar una relación adecuada entre la estructura, la estrategia, los sistemas, el estilo, las capacidades, el personal y, los objetivos superiores de la organización. O, dicho de otra manera, la gestión como la capacidad de articular todos los recursos disponibles.

El ideal ante la lucha contra la burocracia, es la búsqueda de una estrecha relación de movilización de recursos desde los niveles más bajos de la estructura y no desde las exigencias supremas del gobierno central, quién diseña proceso administrativos sumamente ineficientes e influenciados por las recomendaciones de organismos internacionales. Nos referimos a exigencias centradas en adoptar políticas gubernamentales encaminadas a obtener resultados en las evaluaciones mundiales del desempeño escolar, liderazgo y competencias de aprendizaje. El obstáculo está en que esas iniciativas pedagógicas, si se continúan diseñando, programando, aplicando y evaluando desde la cúpula central, en este tipo de sistemas, no llegan a proyectarse en acciones desde y para las escuelas. Porque antes se perdieron en ese largo camino burócrata de exigencias administrativas, normativas y no pedagógicas, que podríamos denominar como una gestión escolar de riesgo.

Una segunda vertiente de gestión escolar, sería una de oportunidad. Hablamos de un proceso que inicie desde la base del sistema, la escuela como eje para el diseño de metas y transformación. En donde la comunidad escolar construya, diseñe sus objetivos y proyectos escolares con la guía del equipo directivo. Así, la evaluación y el progreso de resultados sería sobre sus propios diagnósticos, es decir, la evaluación como un proceso de mejora y autoconocimiento que permita desarrollar y planificar desde una autonomía escolar.

\section{LOS CONSEjOS TÉCNICOS EsCOLARES COMO ESPACIOS DE GESTIÓN, LIDERAZGO, REFLEXIÓN, PARTICIPACIÓN Y ACCIÓN DENTRO DE UNA GOBERNANZA ESCOLAR.}

Todo este esfuerzo estructurado de una nueva gestión escolar abre las puertas para analizar una de las unidad de participación olvidada pero ya existente dentro del sistema educativo mexicano, el órgano colegiado más significativo en los centros educativos, los hoy denominados Consejos Técnicos Escolares (CTE), que nos permite examinar el papel de la autonomía escolar bajo la limitante de la burocracia.

Los CTE, representan la trama organizativa donde los actores más importantes del proceso educativo: Directores, Profesores, Alumnos, Padres de Familia y Comunidad pueden plasmar sus propuestas y tomar decisiones con el objetivo de mejorar las condiciones del plantel educativo este es su principal objetivo utópico-. Lo interesante es que los CTE, tienen su origen como menciona Latapí (2000), desde 1937 cuando la SEP, crea el órgano de consulta del Consejo Consultivo de Enseñanza, que formula un nuevo reglamento para las escuelas e introduce la 
figura de consejo escolar en cada plantel y dichos organismos en sus inicios eran compuestos por colegios de profesores según su especialidad y los consejos consultivos donde se reunían eran denominados reuniones de academia.

Posteriormente se realizaron mas adecuaciones y la creación de organismos alternos. Con la Ley General de 1993, se establecen las disposiciones respecto a la participación de los padres de familia y la sociedad civil en los llamados Consejos de Participación Social en la Educación (CPSE). Además de Consejos Escolares, la Ley promovió la creación de un Consejo Nacional de Participación Social en la Educación o (CONAPASE) y de consejos Estatales y Municipales que es el órgano equiparable al CTE actual. Después de un tiempo de estancamiento y poca difusión de los mismos, en el Programa Nacional de Educación 2001-2006 se reconoce las condiciones poco propicias para el trabajo colegiado. Entonces el asunto de la gestión escolar comenzó a recibir una mayor atención a través de diversos programas, y en la política educativa hacia la transformación de la escuela mediante la participación social en educación con la creación de los consejos de colaboración y la reactivación de los CTE.

Las últimas modificaciones que presentan los CTE es en 2013 y 2019, en el que se reforman el artículo 3o. en sus fracciones III, VII y VIII; y 73, fracción XXV, y se adiciona un párrafo tercero, un inciso d) al párrafo segundo de la fracción II y una fracción IX al artículo 3o. de la Constitución Política de los Estados Unidos Mexicanos, por el que se sientan las siguientes condiciones:

Los Consejos son instancias de participación social en la educación, de consulta, orientación, colaboración, apoyo e información, según corresponda, con el propósito de participar en actividades tendientes a fortalecer, ampliar la cobertura y elevar la calidad y la equidad en la educación básica. (Constitución Política de los Estados Unidos Mexicanos, 2015).

El CTE según sus lineamientos pretende, atender y centrar sus tareas tanto en el nivel básico como medio superior de la educación, en una gestión de los procesos de enseñanza aprendizaje dentro del aula con una gestión escolar a favor de los aprendizajes y la participación social. Su implementación se ha hecho mediante distintos programas hasta llegar al Programa Escuelas de Calidad, con financiamiento del Banco Mundial. Mediante una idea ambiciosa y atractiva para la mejora educativa se incorpora el proyecto escolar, intentando modificar la política de gestión escolar y las dinámicas de trabajo y prácticas de las escuelas. Lamentablemente se olvidó la relación lineal de la gestión escolar y la muy arraigada burocracia de la administración educativa mexicana.

El resultado de esos espacios de autogestión, de participación creativa, de intercambio pedagógico, de búsqueda de soluciones locales, de análisis y diagnostico y sobre todo de desarrollo de un proyecto escolar acorde a las características y necesidades imperantes para la escuela. Es que se han convertido en unos CTE, asfixiados, agobiados, sobrecargados porque funcionan con agendas preestablecidas desde el gobierno central. Las temáticas abordadas son alrededor de temas generales e impuestos para el mejoramiento de la calidad, saturando la agenda con temas técnico-administrativos que además tienen que mostrar evidencias de trabajo y por tanto el tiempo es limitado o nulo para trabajar temas de interés especifico. Estas consecuencias son reportadas no sólo en el nivel de primaria sino también en secundaria y bachillerato.

De esta manera, los profesores se dedican a seguir las agendas impuestas, a contestar las guías que se les presentan en cada sesión y se enfocan en desarrollar las evidencias que se les exigen en 
programas posteriores, es decir una carga administrativa engorrosa. Lo que conlleva a que no encuentran vinculación específica en los aspectos técnicos que se trabajan en los CTE y las realidades educativas diarias. Tampoco representa un espacio en donde puedan encontrar áreas de oportunidad o soluciones creativas para trabajar dentro del aula. Todo lo contrario, ahí se generan, más trabajos administrativos alternos al proceso educativo, lo cual agobia y estresa a los profesores. Finalmente, los directores tienen que presentar largos informes de resultados y un proyecto educativo que es tutelado y no diseñado en su propio centro. La gestión escolar esta dirigida mayormente a los aspectos administrativos con la formulación y exigencias de formatos de rendimiento de cuentas, así como una excesiva normativa. Circunstancias que no permiten una verdadera construcción colaborativa donde participen todos los actores del proceso educativo.

En este punto, tendríamos que reflexionar si tal vez, los países deberíamos mirar un poco a nuestro pasado para encontrar los errores y no volverlos a cometer y de esta manera potenciar los aciertos, rescatando estructuras participativas con las que la comunidad escolar ya esta identificada y después de eso si mirar las experiencias internacionales para no someter estas unidades a procesos administrativos engorrosos y burocráticos.

Es lamentable, que los espacios de participación, que representan la principal oportunidad de una verdadera autonomía, para que la comunidad escolar proyecte sus metas y diseñen sus líneas de acción a lo largo del curso escolar, es solo un procedimiento administrativo, que nunca o pocas acciones se tornan en las prácticas educativas del día a día. Finalmente, las centros escolares terminan con una ausencia de un sistema de objetivos y metas específicas a lograr, por centrarse en una valoración y rendición de cuentas constante.

Hasta ahora, hemos podido dar una pincelada a uno de los espacios de participación en las escuelas mexicanas y, pudimos corroborar que el proceso de colaboración no es un elemento nuevo para los actores educativos. Tejada (1998) coincide en que México ya cuenta con algunos elementos que son determinantes para una innovación del proceso de liderazgo, como la cultura colaborativa y los procesos de auto revisión, planificación, desarrollo y evaluación. Lo cierto es que tiene debilidades, como advertimos en las características de los CTE, en cuanto a ligar los aspectos pedagógicos con los de tipo organizativo, esto debido a las fuertes demandas administrativas del nivel central.

Cuando hablamos del proceso de rendición de cuentas es indispensable girar las miradas a los actores principales en los cuales recae esta responsabilidad y de los cuales depende en gran parte la innovación y un manejo diferente de este proceso de autonomía progresista de gestión. Nos referimos a los directores. En México estos lideres escolares, presentan severas presiones que imposibilitan el cambio o fomentan la continua realización de practicas tradicionales y administrativas, reincidiendo en procedimientos burocráticos de control de rendición de cuentas.

Es así, como el concepto de gestión escolar se ha ido construyendo y avanzando de una percepción técnica, administrativa y financiera a una enfocada en la persona individual del director, es decir a una función centrada en la cultura organizacional, la acción educativa y dentro del ámbito social ya no aislada a una oficina, o por lo menos ese es el ideal de liderazgo escolar.

Para Ball (2016), se esta generando un nuevo lenguaje que reescribe las relaciones entre maestros, directores, ciudadanos y el Estado, que podría mejorar las deficiencias administrativas pero debilita la democracia "la administración, o el liderazgo, como se llama ahora en la educación, es 
un sistema de entrega para el cambio, un método para re cultivar a las organizaciones educativas, y es el punto de apoyo para cambiar las relaciones" (p.1049).

Para México la reforma de 2012-2013, representa el eslabón en donde se otorgo nuevamente a los directores escolares la autonomía y muchas acciones se centraron en el rol de los directores y su profesionalización como figura de liderazgo mediante un plan de autonomía de centro, pero bajo una evaluación estandarizada con mecanismos de evaluación externa y autoevaluación, además de un sistema de rendición de cuentas de los docentes. Por lo tanto, un enfoque gerencialista de reforma educativa y el resultado obvio, fue que se perdió el enfoque académico de la autonomía, diluyéndose dentro del ritmo de la constante exigencia de resultados y rendición de cuentas.

Al mismo tiempo el gobierno, aprueba una "serie de políticas que socavan la autonomía escolar, especialmente la dimensión pedagógica de ésta. Ha promovido un ambicioso programa de competencias básicas, que favorece la recentralización del currículo e introduce exámenes estandarizados de alto riesgo al final de cada etapa" contradiciendo el ideal de autonomía escolar y debilitando "la capacidad de los maestros para determinar autónomamente qué y cómo se debería enseñar en las escuelas" (Verger, Curran, Marta, y Parcerisa, 2015, p. 689). Con un doble discurso se realizan programas para la organización y funcionamiento del centro, con acciones que promueven el proceso educativo de autonomía. Pero en ningún momento se reduce la carga administrativa.

Evidentemente, los centros deben dar prioridad a la finalidad educativa en sus aspectos pedagógicos pero también son impredecibles dentro de la gestión, todos aquellos procedimientos administrativos y económicos, que dan operatividad formal y financiera al proceso. Es cierto que, gran parte de esa gestión administrativa se traduce en una práctica burocrática general, en tanto que, la gestión económica, requiere de un proceso de rendición de cuentas que siempre ha implicado el control y la supervisión de los presupuestos, el inventario y documentación excesiva.

Por lo tanto, para hablar de una verdadera autonomía de la escuela, tendríamos que hacer referencia a una autonomía académica, administrativa y financiera en la que se debería ampliar y profundizar la participación de la comunidad escolar, con lideres que tengan las habilidades para manejar equilibradamente esos aspectos de la gestión escolar.

\section{LA RENDICIÓN DE CUENTAS, EL FENÓMENO QUE OBSTACULIZA LA PRÁCTICA DE LA AUTONOMÍA EDUCATIVA.}

Con el compromiso de garantizar la calidad del desempeño escolar, se están adoptando nuevas formas de gobierno y por lo tanto de administración y gestión, pero sobre todo de rendición de cuentas. En una búsqueda por legitimar los controles de calidad y de resultados, sobre todo cuando se habla de presupuesto público de los sistemas educativos siempre se es más estricto y cauteloso. Normad y Verger (2015), describen esta rendición de cuentas o accountability como una más, de las múltiples herramientas de la NGP, que se encuentra dentro de una lógica de gobernanza escolar centrada en el desempeño y las relaciones auditables y performativas. En palabras de Falabella (2015), un poder a la distancia, lo que también se conceptualiza como el Estado evaluador.

Regresando a aquellos discursos que se conforman de imposiciones mundiales para y por la educación expuestas anteriormente, Rizvi y Lingard (2013) consideran que, la OCDE ha sido un factor clave en la construcción de un espacio generalizado de medición educativa como elemento 
importante de la nueva gobernanza, a través de los indicadores y mediante su Programa para la Evaluación Internacional de Alumnos (PISA). Reflexionan acertadamente en dos aspectos que considera este organismo y, que son trascendentales en la conformación de esa retórica global. El primero se refiere a cómo ese elemento de medición, representa la medida más legitima y exacta de rendimiento educativo internacional. En segundo término como promueve la competitividad internacional y esta a su vez, ha determinado factores de la calidad de la educación medida por dicho estándar.

A la luz de estos argumentos, cobran sentido la multiplicidad de instancias externas de naturaleza evaluadora que están surgiendo en el panorama internacional, que sin considerar elementos del contexto especifico, emiten análisis de esos resultados con propuestas y recomendaciones para lograr el éxito. Justamente de esta manera, se van perdiendo figuras importantes de evaluación local tal y como se pone de manifiesto en el trabajo de Espejo y Calvo (2015), en el que se plantea cómo algunas competencias elementales de la supervisión están siendo usurpadas por agencias de evaluación privadas y evaluadores externos. Se vuelve natural que el grupo directivo de supervisores, inspectores, jefes de enseñanza, etc. se preocupen más por la aplicación de programas dirigidos a mejorar los resultados de esas pruebas estandarizadas o dar seguimiento a los lineamientos de organismos internacionales que por participar y ser guías en la cohesión de grupos colaborativos pedagógicos, centrándose en procesos administrativos de entrega de resultados.

Nos referimos a un proceso que implica una doble rendición de cuentas del que hace énfasis Falabella (2015). Por una parte, algunos de los establecimientos deben rendirle cuentas al mercado, dando respuesta a la demanda de las familias y así captar mayor matrícula. Por otra parte, las instituciones escolares deben rendir cuentas al Estado por el cumplimiento de estándares y metas de desempeño. El autor, subraya que se trata de una lógica empresarial basada en principios de competencia, riesgo, cumplimiento de metas cuantificables e incentivos ligados a resultados, elementos que son de carácter puramente del mercado competitivo pero que se han filtrado a un bien público al servicio de todos los ciudadanos.

Rendir cuentas en este contexto significa, "dar pruebas de un uso eficiente y eficaz del dinero público" (Kehm, 2011, p.29.) y no de su distribución para el logro de objetivos centrados específicos del centro educativo. Esa gestión orientada a resultados para Falabella (2015) esta nutrida por la teoría de las escuelas eficaces. Los supuestos de la política, son que los establecimientos tienen autonomía en los modos de efectuar su gestión y, a la vez, son responsables de su desempeño y deben rendir cuenta por ello, hablamos de una rendición de cuentas con responsabilidad individual.

Existen dos conceptos importantes en términos de modelos de organización que dan estructura al proceso de rendición de cuentas, uno de ellos es la calidad y otro la delegación de facultades o autonomía, en donde, "los gestores se centran en los resultados, no en el proceso y, por tanto, son responsables de los éxitos o fracasos de sus organizaciones" (Mayne y Zapico 2000, p.30); podemos entonces hablar de responsabilidades individuales basadas en lineamientos sobre exigencias de eficacia y productividad.

Es verdad que la calidad no sólo es un asunto que preocupe al ámbito educativo, es un concepto que mundialmente está activo en diferentes áreas. Álvarez (1998), lo concibe como, un impulso humano innato para la superación y la consecución de mejores resultados. "El concepto de calidad ha entrado a formar parte de nuestra cultura social así como otras palabras, igualmente 
importantes (democracia, libertad, igualdad...)" (p.9). Pasando desde los japoneses y americanos, que son sus creadores, llegado en los ochentas a Europa y posteriormente escucharse en Latinoamérica para finalmente hacerse de uso general "en boca de políticos, empresarios, directivos y profesionales de cualquier actividad e, incluso, en boca de los ciudadanos en general" (p.10). Para consecutivamente ser parte de las propuestas políticas de administraciones públicas y dentro de estas el ámbito educativo.

Estas prácticas en función de la calidad y la autonomía, implican que se deleguen acciones y decisiones al personal. Este hecho representa la necesidad inmediata de un informe detallado, actual, fiable, organizado sobre los resultados de las diferentes áreas de gestión para tener la certeza de que se esta trabajando conforme los propósitos establecidos y para el logro de una excelencia dentro del servicio público. De esta manera, es indispensable una clara y buena información de resultados. Esto es, la medición como: "un elemento clave de la gestión de calidad total y de las iniciativas de mejora de la calidad. Todas las discusiones sobre gestión de la calidad y el aprendizaje apunta a la importancia de la medición y el seguimiento de resultados" (Mayne y Zapico, 2000, p.32). Sin embargo, el autor asume que es indispensable que la gestión por resultados, continúe perfeccionándose para no ser solamente un compendio de informes administrativos de resultados.

Inclusive en "determinadas medidas de rendición de cuentas se han fortalecido el control y la presión sobre la profesión docente" (Normad y Verger, 2015, p.603). En diversas reformas aplicadas en los diferentes países, en donde se ha creado un clima de estrés y tensión laboral que no permite que los procesos de organización se desenvuelvan con natural participación y se enfocan en el perfeccionamiento administrativo de documentos, procesos y evaluación. En esta misma línea crítica, Álvarez (1998) asume que la problemática reside en las metas sumamente inalcanzables y admirablemente atractivas que invaden los discursos políticos en el ámbito educativo para sustentar sus reformas educativas y lo denomina discurso a la no calidad.

Un ejemplo de esto es el que presentan Collete y Antoni (2016), en donde analiza el modelo neoliberal en escuelas del Reino unido y, concluyen que la excesiva responsabilidad que asumen las direcciones; la creciente complejidad y exigencia ante tareas de direcciones y docentes; ha llevado a que las tasas de angustia, depresión y/o apatía de los profesionales y alumnado crezcan con el tiempo; y todo esto, es el resultado de que los centros estén altamente vigilados, controlados y supervisados desde las instancias exteriores.

Las políticas educativas están cada vez más determinadas por las exigencias globales que por necesidades locales, como hemos analizado a lo largo de este documento. Rizvi y Lingard (2013) lo ratifica en su análisis de los propósitos educativos, en dónde encuentra que éstos, se han redefinido en términos de un conjunto más estrecho de intereses acerca del desarrollo de capital humano y del rol que debe jugar la educación para satisfacer las necesidades de la economía global y asegurar la competitividad de la economía nacional. Este tipo de políticas somete la labor docente y sus objetivos a medidas estandarizadas de calidad y, por ello, representa un desafío a la autonomía profesional de los maestros (Normad y Verger, 2015).

El resultado es que, este enfoque puede ser poco claro para los directores y docentes al tratar de aplicarlo en sus espacios de acción así que, generalmente la consecuencia es cumplir por rendir cuentas y resultados favorables, por demostrar su trabajo y en muchos casos su estabilidad laboral. Parece que se ha olvidado que estos actores principales tienen las estrategias, la profesionalidad, la experiencia y el corazón para poder actuar acertadamente en cuanto a practicas 
educativas y estrategias en cada una de las aulas. Lo han hecho a través de la historia de los sistemas educativos, han sacado adelante la educación de sus países, pero hemos perdido la fe en ellos y desde las bóvedas más altas, se les quiere manejar como marionetas. Cuando sería mejor soltarles las manos y dejarlos diseñar, planear, hablar, colaborar entre ellos con una visión local y global de una forma colaborativa.

Es claro que, estas reformas educativas llenas de conceptos mundialmente aceptados son rechazados o en el mejor de los casos, asumidos temerosamente por los actores educativos. Se implementan con métodos de control sin tener en cuenta características especificas de cada centro educativo. Se pierde el objetivo y en ese mismo proceso incluso se pierden o difumina la figura de los principales actores educativos. El problema reside en que: "cuando frente a esta visión plural e integradora de los valores de instituciones públicas, se produce una aplicación dogmática de la gerencia, como un paradigma absoluto que remplaza a los preexistentes haciendo tabla rasa de sus valores y marcos institucionales" (Spink et al., 2001, p.104).

De ninguna manera pretendemos desacreditar el concepto de rendición de cuentas, en tanto que es un elemento crucial en términos de la mejora continua y análisis de aspectos oportunos a mejorar. En ese sentido Mayne y Zapico (2000), hace énfasis en la importancia de "una buena información sobre resultados de la gestión, en tanto que, por sí sola no va a reformar el sector público; pero es una herramienta de gestión esencial para lograr con éxito la reforma, y permite a los gestores lograr la excelencia posible y crear una cooperación significativa con el público" (p.34). El seguimiento de resultados, implica también una serie de valores, actuaciones y decisiones dentro de la gestión. Sólo desaprobamos que sea un proceso que fortalezca el control, presión y desajuste de la profesión docente (Ball, 2003, p.11) ó que se establezcan "políticas que someten la labor de los profesores y sus objetivos a medidas estandarizadas de calidad y, por ello, representa un desafío a la autonomía profesional de los maestros" (Normad y Verger, 2015, p.603).

También es verdad que aumentar la capacidad de acción de los directores, maestros y padres de familia, es una táctica para elevar el rendimiento de los alumnos como estrategia de descentralización de gestión escolar que puede tener un impacto muy positivo sobre la calidad de la educación. Si pensamos que la mayor participación y la incidencia, cooperación y sobre todo aceptación de políticas educativas de estos actores, asegura la implementación fluida, consiente, oportuna y directa de acciones centradas en el aprendizaje real. Lamentablemente la realidad muestra que dichos actores están regidos aún por un modelo que privilegia la exigencia de resultados, que esta diseñado desde niveles muy alejados a las necesidades especificas y sobre todo se encuentra cargado de programas por cumplir, evidencias por presentar, informes por realizar y así una lista interminable de requerimientos que genera mayor presión en los centros escolares, dando prioridad a rendir cuentas de resultados que a centrarse en diseñar y llevar acabo sus proyectos escolares.

Es entonces, cuando giramos las miradas, a la importante responsabilidad y no solo en términos de obligación, sino más bien en términos de compromiso y participación de las figuras centrales de las escuelas como agentes vivenciales de los problemas sociales, educativos y económicos de la realidad educativa, para que nos sensibilicemos a entender la imperiosa necesidad de conseguir la verdadera participación, implicación y colaboración de profesores, equipo directivo y padres de familia en la toma de decisiones de los centros educativos para conseguir una rendición de cuentas democrática desde la autonomía escolar. 


\section{CONCLUSIONES}

La historia de las administraciones públicas en el caso de México presenta una transformación de la tradicional burocracia central hacia una gestión enfocada en las responsabilidades y resultados que finalmente se fusionó y se estancó. Posteriormente con un enfoque de la NGP pretende una transición de esa vieja administración hacia organizaciones más flexibles. Pero, es claro que los modelos de institución pública presentan la adopción de paradigmas globales con una descentralización que ha ido conformando la base de diversas reformas dentro del sector público y entre estos el educativo acompañada con un ideal de democracia participativa que aún sigue siendo un reto pendiente.

Evidentemente es indispensable un proceso que reinicie esas estructuras administrativas burocráticas a nivel central que obstaculizan los procesos consultivos y participativos de los centros escolares. Para dar paso a una gobernanza escolar que promueva una intervención democrática pero sobre todo este basada en la acción local como medio para atender, reconocer, diagnosticar y diseñar por y para la escuela, con una renovada cercanía con la comunidad. Apostar por una participación que no inicie desde el nivel central jerárquico de la educación sino desde el eslabón primario que son las escuelas con sus estudiantes, profesores, directivos y comunidad escolar.

El éxito de la gestión escolar reside en la eficiencia de la red de organizaciones que la conforman, la manera en que se movilizan y utilizan los recursos, actividades y resultados que se pretendían en función de los objetivos de la comunidad escolar. Para lograr esa activación de recursos se debe permitir que verdaderamente se involucren en la toma de decisiones. La comunidad escolar tiene que sentirse parte del proyecto de innovación para poder ser cómplice del cambio. Hablamos de una aceptación voluntaria de responsabilidades desde los diferentes niveles de acción de los actores educativos. La participación local, como estrategia de intervención en la detección de problemas, en la toma de decisiones y en el diseño de propuestas. Esto nos lleva a la búsqueda de una democracia en términos de participación educativa

Apostar por la comunidad escolar con la finalidad de encontrar una solidez en las estructuras de gestión debilitadas por la burocracia y las exigencias globales, reside en permitir que aquellos quienes viven la realidad educativa especifica de su contexto, tengan la posibilidad de no sólo e ser simples ejecutores de programas sino también defensores de un proyecto escolar que les afecta de manera totalmente directa y el cuál también pueden elegirlo, protegerlo, renovarlo y evaluarlo conforme a sus necesidades y bajo un compromiso colaborativo. Lo que permite consolidar sociedades más democráticas que tienen en sus manos el recurso educativo para defenderlo.

Entendemos entonces, no solo una participación sino también una responsabilidad comunitaria de espacios para discutir, analizar y proponer. En donde se reconsidere seriamente la compleja interacción entre los procesos administrativos, la pedagogía, el currículo y la evaluación que están dentro de las escuelas y no en un ámbito externo y mucho menos global. Apostando por revertir y encontrar soluciones al deterioro que en términos de estandarización que han perjudicado a la figura de los profesores y personal directivo limitándolos a un papel con escasa intervención que termina por restringir su deseo de implicarse y perseverar en el proceso de transformación educativa. 
En definitiva, tenemos la necesidad imperante de atender todo aquello que no se encuentra programado, sistematizado y estructurado desde los niveles centrales de la educación. Sino al contrario, esta presente en el día a día de las escuelas, que viven, experimentan, lamentan y resuelven continuamente desde la comunidad escolar esas prioridades educativas. Para este fin básico de la educación se necesitan unidades de acción y participación más próximas, más flexibles, más manejables pero comprometidas y con las manos libres para actuar con autonomía bajo una gobernanza escolar democrática.

Finalmente, el potenciar espacios para la toma de decisiones reales y contextuadas dentro del ámbito de la gobernanza escolar, requiere alejarse de decisiones sistemáticas y globales de la estructura central, para poder discutir el qué y para qué de la educación que se imparte en cada centro escolar, así como, el qué y cómo lo pretenden obtener para innovar sus propios procesos educativos. Las voces de estudiantes, docentes, directivos padres de familia y comunidad escolar, son el eje que debe sostener estos debates, porque finalmente son ellos quienes aplicaran todas las políticas y medidas del sistema educativo. Entonces, necesitan encontrar un sentido a lo que realizan para que estén implicados y comprometidos en la búsqueda de objetivos comunes y descubriendo sus propios límites y dificultades que ayudarán a afianzaran las relaciones entre ellos y sintiéndose parte importante e indispensable para el logro de los propósitos del centro. Sin un verdadero compromiso de estos actores, la transformación hacia la innovación no se producirá y terminará siendo lo que hasta ahora ha sucedido, un simple proceso de reproducción, de ejecución administrativa y no de compromiso y participación colaborativa. 


\section{BIBLIOGRAFÍA}

Albi, E., González-Páramo, J., y Casanovas, G. (1997). Gestión Pública. Fundamentos, técnicas y caso. Barcelona: Ariel Economía.

Álvarez, M. (1998). El liderazgo de la calidad total. Madrid: Escuela Española, S.A.

Alvariño, C., Arzola, S., Bruner, J., Recar, M., y Vizcarra R. (2000). Gestión Escolar: un estado del arte de la Literatura. Revista Paidea , 18(4), 15-43.

Astiz, M. (2015). Autonomía Educativa en la Provincia de Buenos Aires, Argentina: comparación entre dos distritos escolares. Una perspectiva Neo- Institucionalista. Educ. Soc., Campinas, 36(132), 647-673.

Ball, S., y Corbalán, F. (2013). Un nuevo escenario para la politica y el negocio educativos. Cuadernos de Pedagogía, (435), 44-47.

Ball, S. (2016). Neoliberal education? Confronting the slouching beast. Policy Futures in Education, 14(8), 1046-1059.

Barzelay, M. (2003). La nueva gestión pública. Un acercamiento a la investigación y al debate de las políticas. México: Fondo de Cultura Económica.

Blanch, J. (2011). Descentralización y autonomía en el sistema educativo en España. El caso de Catalunya. Italian Journal of Sociology of Education, 62(1), 345-350.

Collete, J., y Tort, A. (2016). La Gobernanza Escolar. Madrid: Ediciones Morata, S. L.

Constitución Política de los Estados Unidos Mexicanos, DOF 10-07-15, México 2015.

Davis, G., y Margaret, A. (1992). Escuelas eficaces y profesores eficientes. Madrid: Editorial La Muralla.

Luzón, A. y Torres, M. (2009). La Educación Internacional: Un nuevo escenario entre naciones desde una perspectiva global. En $\mathrm{M}^{\mathrm{a}}$. J. Martínez Usarralde (Coord.), Educación internacional (pp. 15-55). Valencia: Tirant lo Blanch.

Egido, I., Alvarez, A., y Figueiredo, F. (2000). Organización y gestión de los centros educativos en Iberoamérica. Madrid, España: Organización de Estados Iberoamericanos para la Educación, la Ciencia y la Cultura (OEI).

Espejo, V., Calvo, Á. (2015). ¿Contratendencias en la Supervisión Educativa? Las Políticas de Inclusión como Herramienta de Control Formal en Uruguay. REICE. Revista Iberoamericana sobre Calidad, Eficacia y Cambio en Educación. 13(4), 61-78.

Del Castillo, A. (2012). Las Políticas Educativas en México desde una perspectiva de política pública: gobernabilidad y gobernanza. magis, Revista Internacional de Investigación en Educación, 4(9), 637-652. 
Falabella, A. (2015). El Mercado Escolar en Chile y el surgimiento de la Nueva Gestión Pública: el tejido de la Política entre la dictadura Neoliberal y los Gobiernos de la Centro izquierda (1979 a 2009). Educ. Soc., Campinas, 36(132), 699-722.

Kehm, B. (2011). La gobernanza en la enseñanza superior. Sus significados y su relevancia en una época de cambios. Barcelona: Educación Universitaria OCTAEDRO- ICE.

Latapí, P., y Ulloa, M. (2000). El financiamiento de la educación básica en el marco del federalismo. México: Universidad Nacional Autónoma de México.

Normad, R., y Verger, A. (2015). Nueva Gestión Pública y Educación: Elementos Teóricos y Conceptuales para el Estudio de un Modelo de Reforma Educativa Global. Educaşao \& Sociedade, 36(132), 599-622.

Macri, M. (2002). Descentralización educativa y autonomia institucional ¿Constituye la descentralización un proceso abierto hacia la autonomía de las escuelas públicas de Buenos Aires? OEI- Revista Iberoamericana de Educación, (192), 487-504.

Mayne, J., y Zapico, G. (2000). Seguimiento de los resultados de la gestión en el sector público. Perspectivas desde la experencia internacional. Madrid: Instituto de Estudios Fiscales.

OCDE. (2015). Política educativa en perspectiva 2015. Hacer posibles las reformas. España: Fundación Santillana.

Ramió, C., y Salvador, M. (2005). Instituciones y Nueva Gestión Pública en América Latina. Barcelona: Fundación CIDOB.

Rizvi, F., y Lingard, B. (2013). Politicas educativas en un mundo globalizado. Madrid: Morata.

Spink, P., Longo, F., Echebarría, K., y Stark, C. (2001). Nueva Gestón Pública y Regulación en América Latina. Balances y Desafíos. Venezuela: Centro Latinoamericano de Administración para el Desarrollo.

Tejada, F. (1998). Los agentes de la innovación en los Centros Educativos (Profesores, directivos y asesores). Málaga: Ediciones Aljibe.

Verger, A., Curran, M., y Parcerisa, L. (2015). La trayectoria de una Reforma Educativa El caso de la Nueva Gestión Pública en el Sistema Educativo Catalán. Educ. Soc., Campinas , 36(132), 675-697.

Wiseman, W., y Stevens, T. (2017). The impact of the OECD on education worlwide. UK: Emerald Publishing. 


\section{SOBRE LA AUTORA}

\section{Haleyda Quiroz Reyes.}

Licenciada en Educación Básica en el área de Biología y en Diseño Urbano Ambiental. Maestría en Urbanismo y Máster en Estudios Avanzados de Educación en la Sociedad Global. Diez años de Docencia en Educación Básica y tres años de Docencia en Formación y Capacitación de Profesores en Metodologías de la Enseñanza en SEP, México. Actualmente estudiante del Doctorado en Educación por la Universidad de Salamanca y Profesora tutora en el Programa de Tutoría entre Compañeros de la USAL.

Contact information: Universidad de Salamanca, Facultada de Educación. Paseo de Canalejas, 169, Salamanca. Teléfono: 642526224 y correo electrónico: hale_quiroz_reyes@usal.es 